\title{
Developing a conceptual, reproducible, rubric-based approach to consent and result disclosure for genetic testing by clinicians with minimal genetics background
}

\author{
Kelly E. Ormond, MS, LCGC ${ }^{1}$, Miranda L. G. Hallquist, MSc, LGC ${ }^{2}$, Adam H. Buchanan, MS, MPH, LGC \\ Danielle Dondanville, MS, LCGC ${ }^{3}$, Mildred K. Cho, PhD ${ }^{4}$, Maureen Smith, MS, LCGC ${ }^{5}$, \\ Myra Roche, MS, CGC ${ }^{6}$, Kyle B. Brothers, MD, PhD ${ }^{7}$, Curtis R. Coughlinll, MS, MBe, $\mathrm{CGC}^{8}$, \\ Laura Hercher, MS, CGC ${ }^{9}$, Louanne Hudgins, MD, FACMG ${ }^{10}$, Seema Jamal, MSC, (C)CGC ${ }^{11}$, \\ Howard P. Levy, MD, PhD ${ }^{12}$, Misha Raskin, MS, CGC ${ }^{2,13}$, Melissa Stosic, MS, $\mathrm{CGC}^{14}$, \\ Wendy Uhlmann, MS, CGC ${ }^{15}$, Karen E. Wain, MS, LGC ${ }^{2}$, Erin Currey ${ }^{16}$ and \\ W. Andrew Faucett, MS, LGC ${ }^{2}$
}

\begin{abstract}
Purpose: In response to genetic testing being widely ordered by nongenetics clinicians, the Consent and Disclosure Recommendations (CADRe) Workgroup of the Clinical Genome Resource (ClinGen; clinicalgenome.org) developed guidance to facilitate communication about genetic testing and efficiently improve the patient experience. Considering ethical, legal, and social implications, and medical factors, CADRe developed and pilot tested two rubrics addressing consent for genetic testing and results disclosure. The CADRe rubrics allow for adjusting the communication approach based on circumstances specific to patients and ordering clinicians.
\end{abstract}

Methods: We present results of a formative survey of 66 genetics clinicians to assess the consent rubric for nine genes $(M L H 1$, CDH1, TP53, GJB2, OTC; DMD, HTT, and CYP2C9/VKORC1). We also conducted interviews and focus groups with family and patient stakeholders $(N=18)$, nongenetics specialists $(N=27)$, and genetics clinicians $(N=32)$ on both rubrics.
Results: Formative evaluation of the CADRe rubrics suggests key factors on which to make decisions about consent and disclosure discussions for a "typical" patient.

Conclusion: We propose that the CADRe rubrics include the primary issues necessary to guide communication recommendations, and are ready for pilot testing by nongenetics clinicians. Consultation with genetics clinicians can be targeted toward more complex or intensive consent and disclosure counseling.

Genetics in Medicine (2019) 21:727-735; https://doi.org/10.1038/s41436018-0093-6

Keywords: Genetic testing; Genetic counseling; Informed consent; Results disclosure; Rubric

\section{INTRODUCTION}

With the rapid increase in genetic testing and limited number of genetic counselors and clinical geneticists ${ }^{1}$ (hereafter referred to as genetics clinicians), it is not feasible, and potentially not desirable, to recommend that genetics clinicians be involved in all instances of consent for genetic testing and disclosure of results. Instead, nongenetics clinicians, including primary care providers, could obtain consent and disclose results in at least some situations. Many such providers are well prepared to provide such services, although some report that their time and low confidence pose important barriers. ${ }^{2}$ Indeed, the literature documents examples of harms that may occur without adequate training, tools, or support. ${ }^{3-6}$ A recent paper and editorial ${ }^{7,8}$ strongly propose that to transition genetic

\footnotetext{
${ }^{1}$ Department of Genetics and Stanford Center for Biomedical Ethics, Stanford University School of Medicine, Stanford, California, USA; ${ }^{2}$ Geisinger, 100 North Academy Avenue, Danville, Pennsylvania 17822, USA; ${ }^{3}$ Department of Genetics, Stanford University School of Medicine, Stanford, California, USA; ${ }^{4}$ Stanford Center for Biomedical Ethics, Stanford University School of Medicine, Stanford, California, USA; ${ }^{5}$ Northwestern University, Evanston, Illinois, USA; ${ }^{6}$ Departments of Pediatrics and Genetics, University of North Carolina at Chapel Hill, Chapel Hill, North Carolina, USA; ${ }^{7}$ Department of Pediatrics, University of Louisville, Louisville, Kentucky, USA; ${ }^{8}$ Department of Pediatrics, Center for Bioethics and Humanities, University of Colorado Anschutz Medical Campus, Aurora, Colorado, USA; ${ }^{9}$ Sarah Lawrence College, Bronxville, New York, USA; ${ }^{10}$ Division of Medical Genetics, Department of Pediatrics, Stanford University School of Medicine, Stanford, California, USA; ${ }^{11}$ GeneDx, 207 Perry Pkwy, Gaithersburg, Maryland 20877, USA; ${ }^{12}$ Johns Hopkins University, Baltimore, Maryland, USA; ${ }^{13}$ Helix, 3505 West Sam Houston Parkway North Suite 400, Houston, Texas 77043, USA; ${ }^{14}$ Columbia University, New York, New York, USA; ${ }^{15}$ University of Michigan, Ann Arbor, Michigan, USA; ${ }^{16}$ National Human Genome Research Institute, NIH, Bethesda, Maryland, USA. Correspondence: Kelly E. Ormond (kormond@stanford.edu)
} 
testing to a population base, we need to consider, develop, and adopt alternative models for genetic testing consent and disclosure. However, this remains a controversial proposal ${ }^{9}$ and there is debate how best to operationalize new approaches to provide patients with the education and support that each needs and desires when undergoing genetic testing. ${ }^{10}$

In response to our own consideration of these issues, the National Institutes of Health (NIH) funded Clinical Genome (ClinGen) Resource established a Consent and Disclosure Recommendations (CADRe) Workgroup. Taking into account the ethical, legal, and social implications and medical factors (ELSIPlus) associated with genetic testing, CADRe developed two rubrics to facilitate a transparent, conceptual, and reproducible process around consent and disclosure of genetic test results. The CADRe rubrics provide a conceptual framework that genetics experts can apply to suggest a "communication starting place" for ordering clinicians. The communication approach can then be tailored based on patient characteristics and clinical judgment. We propose three possible communication approaches (Fig. 1): (1) traditional genetic counseling (TGC) with a genetics specialist, (2) targeted discussion with an ordering clinician, or (3) a brief communication supported by educational resources. The CADRe recommendations and supporting materials assist clinicians in identifying when there is a reason for a more or less intensive communication process. They are designed to improve the patient experience, promote timely referral and testing, and facilitate the appropriate use of genetic testing. In this paper we outline the process of developing the rubrics and their future recommendations, including formative mixed methods pilot data on issues stakeholders identify as necessary.

\section{MATERIALS AND METHODS}

The development of the CADRe rubrics involved (1) expert consensus workgroup development of the rubrics, (2) a formative survey assessment of the consent rubric among genetics experts, and (3) formative qualitative assessment of both rubrics to ensure inclusion of critical ELSIPlus factors.

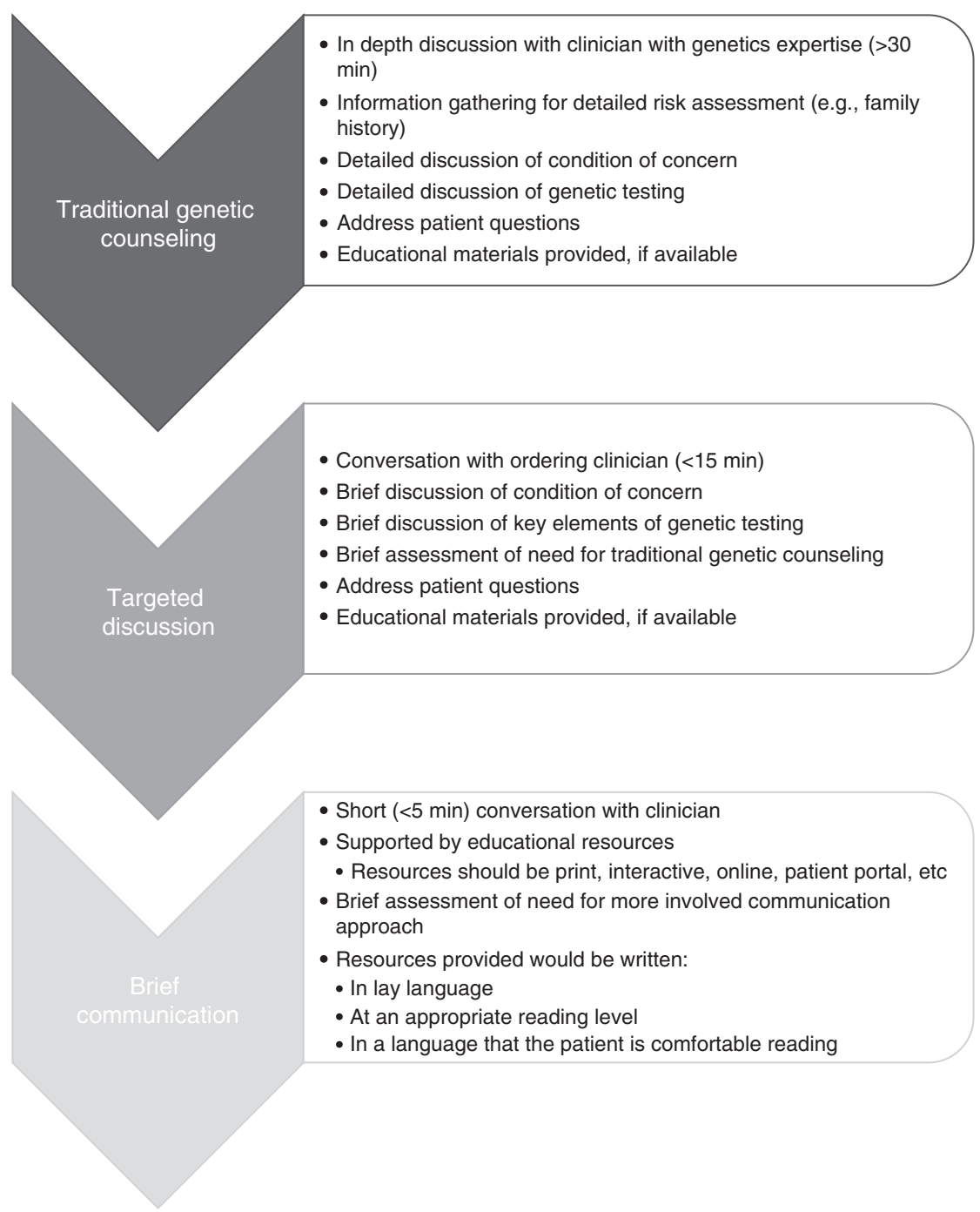

Fig. 1 Description of levels of communication as utilized in the Clinical Genome (ClinGen) Resource Consent and Disclosure Recommendations (CADRe) Workgroup ethical, legal, and social implications and medical factors (ELSIPlus) consent and disclosure rubrics 
Revision and pilot testing of the rubrics are not described in this paper. All study methods were approved by the Institutional Review Boards at Geisinger and Stanford University, and research consent was obtained from all participants. Work in progress was presented to engender discussion around the draft rubrics (European Meeting on Psychosocial Aspects of Genetics 2016, National Society of Genetic Counselors 2015 and 2016; American Society of Human Genetics 2016; American College of Medical Genetics and Genomics 2018).

Expert consensus workgroup development of the draft rubrics Beginning in summer 2014, a 14-member workgroup was established to discuss consent and disclosure of genetic testing results. Monthly discussions centered around the challenges in obtaining consent and disclosing results, whether a tool (in this case a rubric) could be developed to support nongenetics clinicians in triaging referrals for pre- and posttest genetic counseling, what format it should take, and what areas should be considered for inclusion. CADRe came to rapid consensus that the following factors may commonly impact the consent and disclosure process: the clinical indication for genetic testing, the potential emotional burden of the result, patient preferences for information and emotional support, test characteristics (genetic heterogeneity, detection rate, sensitivity and specificity), disease characteristics, and the degree of uncertainty and/or variability in phenotype. Implementation concerns included the notion that posttest genetic counseling is highly dependent on what occurs during pretest counseling, the limited existence and quality of patient-centered educational materials, and the languages in which they are written. Disseminating CADRe recommendations and educating nongenetics clinicians about the rubrics' existence and application will also be a challenge.

In early 2015, CADRe discussed two possible approaches to developing guidance: a conceptual gene/disease-based approach, and an approach based on contextual factors impacting the patient and/or clinician (for example, the patient's anxiety or prior knowledge, test indication, provider knowledge and comfort level). Ultimately, CADRe prioritized a gene/disease-based approach for the rubrics because we felt the contextual factors fell under the purview of clinician judgment and the patient-provider relationship. CADRe created a rubric for consent (Fig. 2) and a rubric for disclosure of genetic test results (Fig. 3). Four key areas were ultimately included (Fig. 2, working definitions): (1) Complexity of testing: communication may require more nuance for patients, or the ordering and interpreting process may be beyond the expertise of an average nongenetics clinician; (2) Increased risk of adverse psychological impact specific to the genetic testing process: patients should weigh these issues as part of the decision-making process around genetic testing, and increased support at both consent and disclosure is warranted; (3) Significant risk for near-term mortality: conditions that have a higher chance of imminent death, for example, should minimally have a targeted discussion as part of consent; (4) Clinically complex management: patients should be aware of the potential for complex management if the results are positive, particularly if the management is onerous

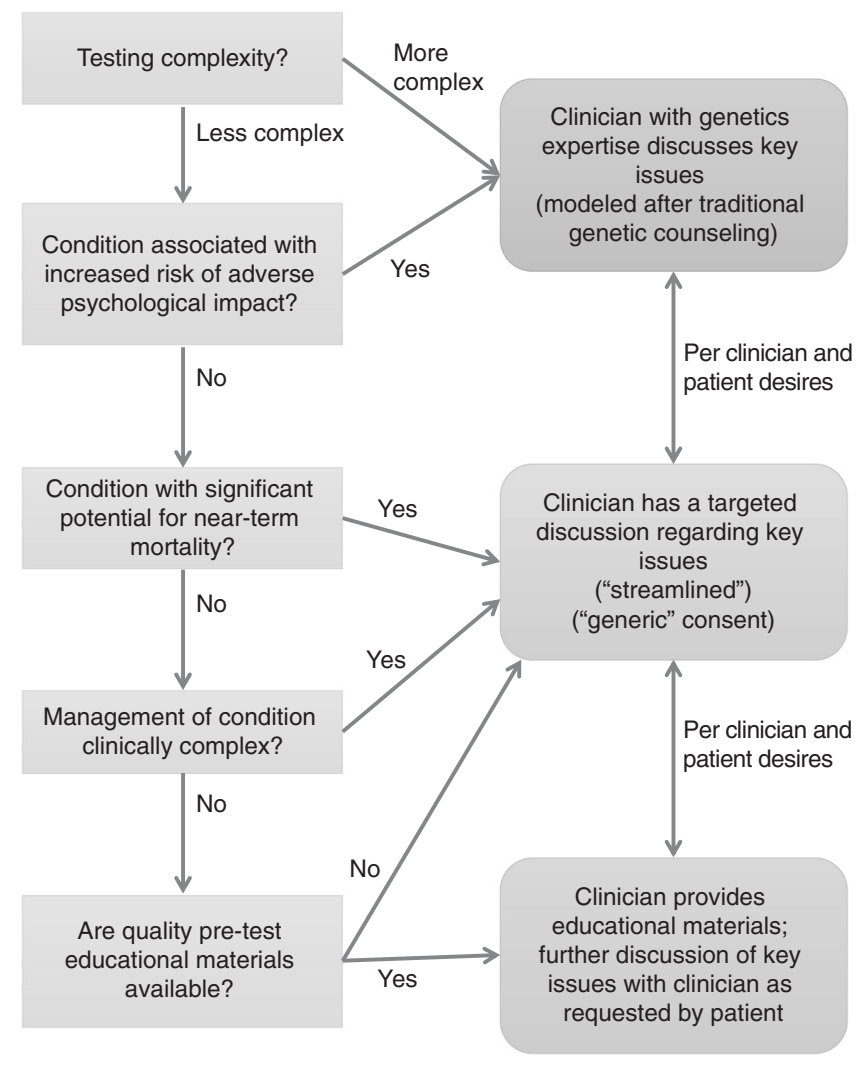

Fig. 2 Consent and Disclosure Recommendations (CADRe) Workgroup ethical, legal, and social implications and medical factors (ELSIPlus) consent communication rubric. Working Definitions: Complexity of testing: A test's complexity is assessed according to the following elements: incidental finding risk, variant of unknown significance risk, clinical validity, residual risk, and indication. At least two areas need to be more complex for the overall test to be considered more complex. (See the text table). Increased risk of adverse psychological impact: Evidence (publication [s] of acceptable rigor) that the particular condition is associated with increased pretest or posttest risk of adverse psychological outcomes (e.g., depression, anxiety, distress, coping concerns, or suicidal ideations). This risk can be for tests that detect pathogenic variants, negative tests (e.g., with evidence of survivor guilt), or both. A theoretical concern about adverse psychological outcomes of a test without supporting evidence is insufficient to meet this criterion. Significant risk for near-term mortality: Condition is associated with risk of sudden death (e.g., dilated cardiomyopathy, Marfan syndrome). Two criteria must be met: (1) the Actionability Workgroup has scored the condition as having a severity of 3 , and (2) CADRe Workgroup determines that the risk is near-term. Clinically complex management: Identification of a pathogenic/likely pathogenic variant would lead to (1) the need for a discussion between the patient (or family) and clinician to develop a detailed or complex clinical plan; (2) interventions that carry significant patient burden (e.g., longitudinal multidisciplinary care, significant impact on daily functioning (e.g., dietary restrictions or daily medication requiring monitoring); or (3) interventions that involve substantial morbidity/mortality risk (e.g., surgical interventions). Quality educational materials: The CADRe Workgroup or ordering clinician has identified material(s) they deem to be written in lay language, broadly accessible, culturally appropriate, and to sufficiently cover the relevant consent or disclosure issues

or knowledge of it may change the patients' testing decisions. The availability of quality educational materials for patients and clinicians is key to meeting the needs of these stakeholder groups, and as such this served as a final determinant of the recommended communication approach. 


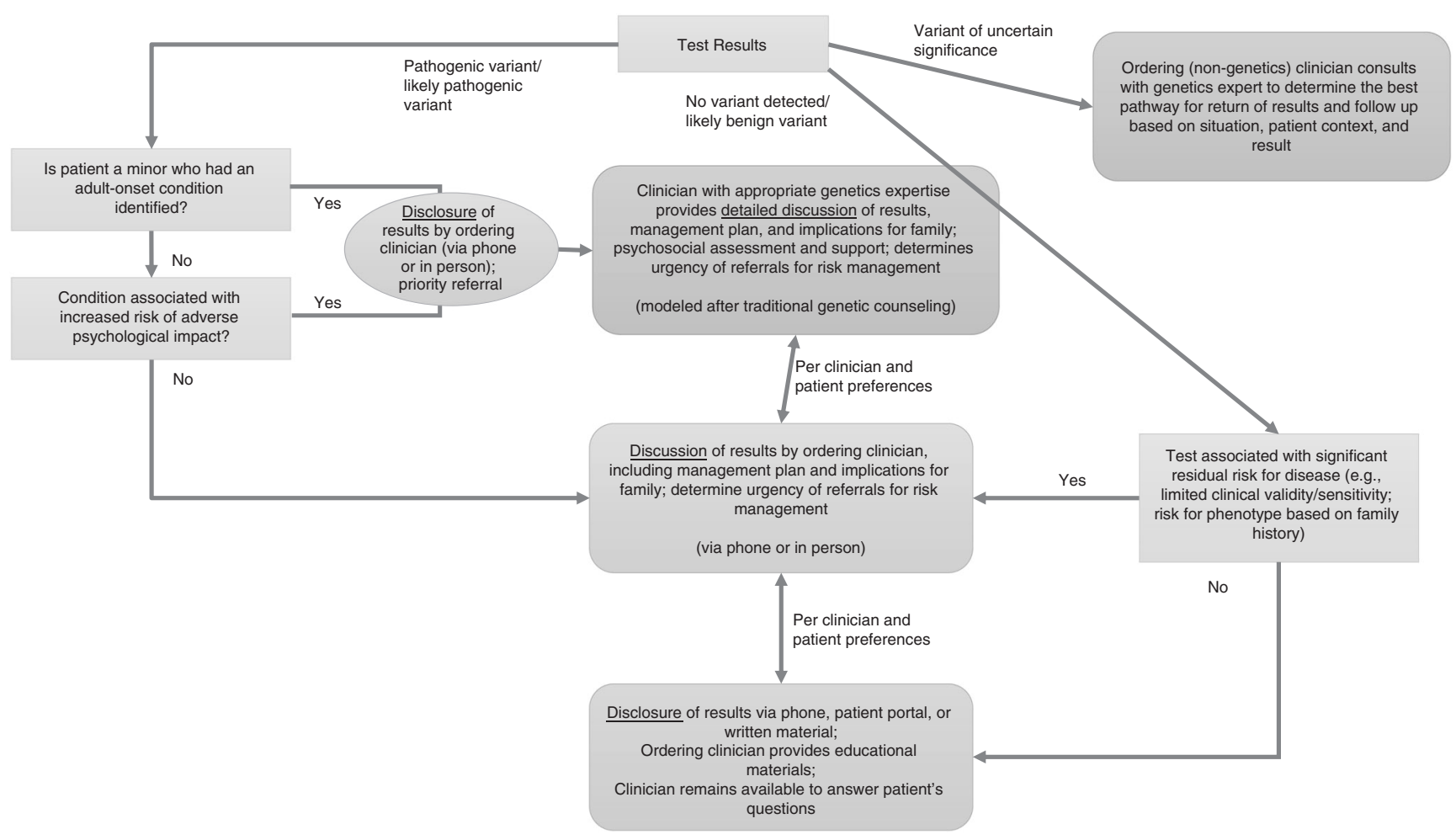

Fig. 3 Consent and Disclosure Recommendations (CADRe) Workgroup ethical, legal, and social implications and medical factors (ELSIPlus) results disclosure communication rubric

\begin{tabular}{|c|c|c|}
\hline Element & More complex & Less complex \\
\hline $\begin{array}{l}\text { Incidental } \\
\text { finding } \\
\text { risk }\end{array}$ & Higher & Lower \\
\hline VUS risk & Higher & Lower \\
\hline $\begin{array}{l}\text { Analytic/ } \\
\text { clinical } \\
\text { validity }\end{array}$ & $\begin{array}{l}\text { Lower sensitivity/ } \\
\text { specificity/PPV/NPV }\end{array}$ & $\begin{array}{l}\text { Higher sensitivity/specificity/PPV/ } \\
\text { NPV }\end{array}$ \\
\hline $\begin{array}{l}\text { Residual } \\
\text { risk }^{a} \\
\text { Indication }\end{array}$ & $\begin{array}{l}\text { Higher diagnostic } \\
\text { testing when diagnosis } \\
\text { is unknown }\end{array}$ & $\begin{array}{l}\text { Lower population screening, } \\
\text { carrier screening, confirmatory } \\
\text { testing for known clinical } \\
\text { diagnosis, known familial variant }\end{array}$ \\
\hline
\end{tabular}

VUS variant of uncertain significance

aspecific to the test or a population, not to a particular patient

\section{Formative survey assessment of the consent rubric among genetics experts}

A formative survey was developed by several members of CADRe $(\mathrm{AB}, \mathrm{WAF}, \mathrm{MH}, \mathrm{KO})$. The survey provided a description of the CADRe project goals, definitions of the communication levels that were proposed, and the intended use of the consent rubric. We utilized 8 clinical scenarios that focused on a specific gene and posed 2-4 different clinical indications for testing. Survey respondents (genetics experts) answered up to 5 scenarios, chosen based on their clinical expertise to complete questions for genes associated with cancer $(M L H 1, C D H 1, T P 53)$ or pediatric conditions (GJB2, OTC; $D M D)$. They also answered scenarios for two genes for which there is broader agreement about the approach to consent: HTT-the Huntington disease test (an indepth discussion during consent is standard) and CYP2C9/ VKORC1-a pharmacogenomic test for which the consent discussion is presumed to be brief. Each gene and testing indication was selected on the basis of several of the principles highlighted by the rubric, and as an example of a commonly used genetic test. For each gene, the survey summarized the CADRe rubric recommendation and rationale, then asked each respondent to select the level of communication they thought was most appropriate for each indication for testing (regardless of the recommendation) and to justify their recommendation (see Supplemental Fig. 1 for example). Exposure to the CADRe rubric recommendations and rationale was intentional, as the goal of this evaluation step was to determine whether the rubric was a useful and relevant tool in assessing levels of communication for patients in different scenarios.

We used an exponential nondiscriminative snowball sampling method ${ }^{11}$ to recruit as large a sample of genetics experts as possible in as wide a range of clinical contexts as possible. The snowball sampling started with members of the CADRe Workgroup, who recruited genetics colleagues to complete the survey between March 24 and April 21, 2016. Participants completed the survey anonymously through online survey software (SurveyMonkey). Descriptive data were analyzed using SPSS version 24 .

\section{Formative qualitative assessment of both rubrics}

We conducted a combination of telephone interviews $(N=23)$ and 10 focus groups $(N=54)$ individuals) between April and 
November 2016 to obtain responses towards the CADRe rubric from nongenetics clinicians, genetics clinicians, and patient/ family stakeholders. We recruited from geographically diverse areas of the United States (Pennsylvania, North Carolina, Illinois, and California) using targeted Facebook advertisements, informational flyers, and word of mouth. Condition support group listservs were also used to recruit patients and family stakeholders. Participants in focus groups were offered a $\$ 50$ honorarium for their time; interview participants were offered a \$20 honorarium.

Clinician participants included a range of genetics clinicians (medical and laboratory geneticists, genetic counselors) and nongenetics clinicians (primary care clinicians, OB/GYNs, pediatricians, neonatologists, cardiologists, pathologists, and oncologists) drawn from academic medical settings, integrated healthcare systems (e.g., Geisinger and Kaiser Permanente), and community healthcare settings, representing urban, suburban and rural healthcare provision. Focus groups of healthcare professionals were conducted, when feasible, with at least two members of the research team in attendance to moderate the focus group $(\mathrm{AB}, \mathrm{MC}, \mathrm{WAF}, \mathrm{MH}, \mathrm{KO}$, MS). With the goal of collecting as many viewpoints as possible, interviews were offered as a backup option due to the complexity of scheduling focus groups, and were conducted by three individuals ( $\mathrm{AB}, \mathrm{MH}, \mathrm{KO})$.

Patient/family stakeholder focus groups included individuals $>18$ years old who underwent or were considering genetic testing for a Mendelian condition, or a family member of such an individual. We excluded individuals who were non-English speaking or with cognitive impairments.

The discussion guides were developed by a subset of the researchers ( $\mathrm{AB}, \mathrm{MC}, \mathrm{WAF}, \mathrm{MH}, \mathrm{KO})$ to address participants' experiences in genetic testing, their sense of the ideal consent and disclosure process, their responses to the consent rubric and clinical examples of its recommendations.

Focus groups and interviews were audio-recorded and transcribed, with identifying information removed. Conceptual summary notes were taken to supplement the transcript analysis. Analysis was performed using Dedoose ${ }^{12}$ version 7.6.18. A codebook was developed iteratively after review of all transcripts $(\mathrm{KO})$, and refined through discussion among key members of the qualitative research team $(\mathrm{AB}, \mathrm{MC}, \mathrm{DD}, \mathrm{WAF}$, $\mathrm{MH}, \mathrm{KO}$ ). For increased objectivity, all interviews and focus groups were coded by a single individual (DD) who was not involved in either the development of the rubrics or the conduct of the interviews or focus groups.

\section{RESULTS}

\section{Online survey results}

Sixty-six genetics clinicians completed a sufficient number of scenarios for inclusion in the analysis. Over half $(57.3 \%)$ of respondents had fewer than 10 years of clinical experience. Practice areas represented prenatal (39\%), pediatric $(39 \%)$, adult $(2 \%)$, biochemical $(8 \%)$, and cancer genetics $(41 \%)$. To maintain anonymity, we did not collect other demographic data.
Supplemental Table 1 summarizes the survey responses. In $20 / 26$ pairings, more than $50 \%$ agreed with the communication level proposed by the rubric. There were three areas where there was disagreement, with participants typically recommending more intensive communication: (1) Cancer genetics scenarios when the testing indication was an unaffected individual with a family history but no affected proband who had undergone testing; this was due to the probability that a negative test result could be a false negative. (2) Carrier testing in an unaffected female, particularly for $\mathrm{X}$-linked conditions (DMD, OTC), due to the psychosocial impacts and potential impact on the woman's health. (3) Familial variant testing, due to the higher chance of a positive result. When asked how best to handle multigene panel testing, $68 \%$ said "if a gene on the panel is categorized as TGC, the panel as a whole should be categorized as TGC," $16 \%$ said "the panel should be categorized in the approach that is appropriate to the majority of genes on the panel," and $18 \%$ indicated through comments that a condition- or indication-specific approach should be taken (7\%), that panels should always include TGC due to complexity (4\%), or that they had no opinion (5\%).

Not only was the specific gene influential in determining a recommended communication level (Supplemental Figure 2), but test indication was also a factor:

"I think the probability of a result differs ... therefore I think they should be treated differently. The stakes ... and the emotions involved are different."

"I think patients are in a different frame of mind and have different goals when in the circumstances of these various testing indications. These will also impact how familiar the patient is with a disease, what stigma they may perceive, and what misconceptions may need to be addressed."

\section{Qualitative data}

We completed 3 patient/family stakeholder focus groups $(N=$ 11) plus 7 individual interviews, 4 nongenetics clinician focus groups $(N=19)$ plus 8 individual interviews, and 3 genetics clinician focus groups $(N=24)$ plus 8 individual interviews (Supplemental Table 2). Focus groups averaged $90 \mathrm{~min}$; interviews averaged $30 \mathrm{~min}$. Table 1 includes illustrative quotations for the primary themes.

\section{Patient and family stakeholder perspectives}

Nineteen patient and family stakeholders (hereafter called patients) represented personal or family experience with cancer (primarily breast and colon cancers), cardiology (primarily long QT), fragile X, Huntington disease, and ancestry testing. Overall, patients prioritized a "quick" and focused consent process that is easy to assimilate and understand. Patients desired TGC when there is a higher chance of a positive result, higher penetrance, greater severity, a significant treatment 
Table 1 Illustrative quotations

Topic

Reasons for a "higher" level of communication:

Disease reasons

Reasons for a "higher" level of communication: Test complexity/logistics

When to see a genetic counselor: Results Disclosure timing

When to see a genetic counselor: Disclosing negative results

Evolving thoughts about communication levels

\section{Quotation(s)}

"I think sometimes conditions that are more involved like Li Fraumeni syndrome, VHL, which I don't do much VHL testing anymore, but... Peutz-Jegher syndrome if I'm really suspicious of that, that's an incredibly involved condition, you know, especially things where you would then second-step ordering to test your kids right away, that's a lot more involved, so I feel like those are ones that I would put in time."-Genetics clinician

"...the biggest reason would be the complexity, you know, folks coming with multiple different types of cancers. It's a little more easy if someone comes in and says it's mainly they're concerned about breast cancer. So for like this particular gentleman, he had multiple family members with various types of cancers, colon cancer, breast cancer, melanoma, so it's kind of hard to target what you would want to order."-Nongenetics clinician

"Well, I just kind of feel like if you get it before, you know, it may be a non-issue. If you know the results of the test and...I was just thinking there may be follow... if you get it before then you get the test back and then you have follow-up questions, there wouldn't be the opportunity for that, but if you can talk with your clinician, have some kind of baseline information beforehand, and then get your test results back, and then you have follow-up questions that can go more in depth with the genetic counselor, I just think it's wiser to have it afterwards." - Patient

"Actually those [with a negative genetic test but clinical features or a diagnosis] are the ones that I think actually benefit more from a traditional genetic counseling session because the management is not as clear cut as following some guidelines, right, now you're not basing your guidelines on having a mutation, it's based on the family history, which takes a little bit more interpretation arguably than a, you know,

mutation that's very clear cut and a gene like one of those mismatch repair genes. So for those patients I do somewhat feel like a more in-depth discussion with a clinician would be beneficial." - Genetics clinician "If the test is negative...I mean I still think that's a bit...I still think that they need to understand that they, you know, meet the clinical criteria and even though the testing result was negative it could just be that they have a, you know, variant that wasn't identified or, you know, something that was outside of the testing methodology so that it doesn't take away their risk and their family risk, so to me that's still fairly complex. I guess I'd still stick with the traditional genetic counseling." - Genetics clinician "I've done testing and counseling kind of both ways for like say the same condition, so I've got an affected patient and it's indicated that they have testing and I do the quick... quick... quicker genetic counseling session upfront and then we do more of a detailed analysis with the doctor on the back end, for pathogenic or VUS or negative on the back end, that works out just fine. If you flip it and you do the more detailed upfront and then you call them up with a negative result, that's perfectly fine too, but if there is more of a complex discussion at some point I think it's beneficial. So for some conditions it's certainly better on the back end because there's more management recommendations involved and like, to be honest, for cardiac, that's actually now how it's kind of preferred to be done, where quick explanation upfront, test, and then because the management is so complex there's something more detailed on the back end."-Genetics clinician

VUS variant of uncertain significance

burden, or a pre-existing clinical diagnosis. They also preferred TGC when there is an at-risk pregnancy and associated anxiety. For people at $50 \%$ risk for a familial variant, there was recognition that patients had diverse views. Some may already be familiar with the condition and have made a testing decision, while others may desire more information to decide whether to be tested. In particular, if patients were anxious, many found the information and psychosocial attention from TGC useful; patients who were not already anxious expressed "not wanting to be made anxious" in advance of results.

Patients prioritized the following in the consent process: what you are testing for, why you are testing for it, what would change based on the test results, and insurance implications. Many perceived that their clinicians discussed genetic testing as a medical recommendation rather than an optional test.

Nearly all patients (17/19) felt that if they could only see a genetic counselor at one point in the genetic testing process, they preferred doing so for result disclosure as long as this did not slow down the time frame to obtain results or to get specialist referrals for management and surveillance. Patients stressed that TGC should be recommended for disclosure of positive results to help patients learn about the complexities of their test result. Finally, for negative results, while some patients and families still agonize over them, most patients felt that all you really need is education materials clarifying what they still need to do/think about. 


\section{Nongenetics clinician perspectives}

Nongenetics specialists $(N=27)$ reported generally feeling comfortable with the informed consent process for what they considered "straightforward test ordering" within their specialty, and return of negative results in these areas. Factors that influenced referrals for pretest TGC included (1) logistical concerns, including institutional requirements, the complexity of test ordering, the case complexity, how common the referral indication is, and the length of wait for a genetic counseling appointment; (2) counseling needs of anxious patients; and (3) the likelihood of a positive result. Because most specialists focus care on individuals, many reported that cascade testing and reproductive recurrence risk counseling is beyond their scope. Regarding key components for genetic testing consent, the nongenetics providers focused on setting expectations for why they are doing the test, likelihood of a positive result, test limitations, and whether results would change medical management. They perceived the consent process differently if clarifying a diagnosis versus offering a predictive test.

There was variation among clinicians regarding how they handled results discussions. Some reported conducting the initial disclosure, primarily due to time and access issues, and then referring for TGC. Several nongenetics participants stressed that providers in their specialty (oncology, neurology) had expertise in delivering bad news, and that for their patients, a genetic test result was often not the worst health news they had received, and this made them comfortable with the emotional aspects of results delivery.

\section{Genetics clinician perspectives $(\boldsymbol{N}=32)$}

Genetics clinicians' $(N=32)$ perception of the appropriate level of communication was influenced by patients' prior knowledge and anxiety level, chance the test will be positive, and whether and how the test would change medical management. Setting patient expectations was seen as a key aspect of consent. Most genetics clinicians felt more comfortable, for example, using a targeted discussion for consent if the patients already knew a fair bit about the condition or had a clinical diagnosis.

Nearly all genetics clinicians expressed initial conflict about the targeted discussion and educational materials approach to consent, often sharing anecdotes from their own practice. This was expressed as frustration about "cleaning up the mess" at disclosure if the consent process "is not handled well," or if the correct test is not ordered. Despite this, most genetics clinicians reached consensus by the end of the discussions that their specialist skills were most useful in the results disclosure setting because that is where many errors can be made by nonexperts (e.g., residual risks, limits of testing) and the education can be targeted to the results and patient response. Interestingly, there was more comfort with a targeted consent discussion about exome testing, acknowledging that result disclosure is the harder part, but with single gene/panel it was sometimes seen as the reverse.

\section{DISCUSSION}

The CADRe rubrics were generally well received by all three groups in our formative mixed methods research. All aspects of our assessment identified patient pretest anxiety and knowledge, the likelihood of a positive test result, and the need to discuss family implications as relevant to triaging communication levels. Nongenetics providers and genetics clinicians identified logistical barriers in test ordering and interpretation, including test complexity and factors such as residual risk for negative results. Patients and genetics clinicians also identified factors related to disease phenotype and treatment as influential. Regarding negative genetic testing results, the major concern of genetics professionals is that patients should be made aware of the limitations of genetic testing.

As such, the recommended communication level could vary depending on the comprehensiveness of testing and implications regarding residual risk. For example, after a negative multigene panel that broadly covered the differential, educational materials may be adequate. If only a handful of the relevant genes were tested, a targeted discussion that also functions as a consent for follow-up testing might be appropriate. When a genetic diagnosis confirms an existing clinical diagnosis, we propose that minimally a targeted discussion should occur to emphasize the importance of family disclosure and cascade testing and address the psychosocial needs of families, which many nongenetics providers feel is outside of their comfort and scope of practice. While families expressed a desire for TGC in this context, we assume that a clinical diagnosis includes a management discussion by the diagnosing clinician, and thus the discussion of genetic testrelated issues will occur separately. Finally, if a pathogenic genetic diagnosis is unexpected, TGC can provide an opportunity for psychosocial support toward adaptation, indepth education, and family cascade testing.

Our stakeholder groups approached consensus that genetics clinicians should play a more critical role at the time of results disclosure rather than consent. We acknowledge this is a potentially controversial recommendation, because there has been an historical emphasis on pretest genetic counseling. In the early days of genetic testing and counseling, genetic testing was often predictive, frequently complicated, and sometimes indirect, using methods such as linkage analysis. ${ }^{13,14}$ Provider and patient knowledge about genetic testing was limited. Testing relied on a well-based differential diagnosis, and test selection and cost were common barriers. Finally, patients undergoing genetic testing classically came from families with a significant lived experience of the condition, and often had personal expectations of whether they had inherited the condition. This frequently led to a significant decision-making about whether to undergo genetic testing, making the informed consent process critical.

Encouragingly, other recent work supports the strategy of moving the focus of TGC from consent to results disclosure and ensuring that consultation with genetics experts will be available and prioritized for more complicated cases. First, there is research in cancer genetics around "genetic counselor extenders"15 with a team approach where a nurse "extender" 
provided targeted discussions and pretest consent in cases that are considered more straightforward risk assessments. Lieberman et al. ${ }^{16,17}$ presented qualitative data from a population screening program for $B R C A 1 / 2$ in Israel that utilized written education materials for pretest consent. Most patients found the streamlined consent process acceptable, and some found TGC created unnecessary anxiety. Finally, Mayo Clinic presented a brief review of their experience using a streamlined written informational approach to exome testing. ${ }^{18}$

For this proposed model to meet patient stakeholder needs, ordering clinicians must evaluate and triage patients for characteristics that warrant a change to the recommended communication level (see Box 1). Patients who desire more information or support, feel unsure about whether genetic testing is right for them, have limited genomic or health literacy, ${ }^{19}$ or are anxious ${ }^{20}$ should be strongly considered for pretest TGC, and may always request it. In contrast, knowledgeable patients, including those who have previously undergone genetic testing, may find educational materials and a brief discussion with a nongenetics provider sufficient. Clinicians may need education, curbside consultations, and support in triaging patients appropriately, and we see this as an important area for collaboration between genetics and nongenetics specialists and for future research.

Second, nongenetics providers need to understand the basic aspects of genetic testing (including test ordering logistics) and the key concepts for consent. Based on our study data and health literacy research, we propose that components of a targeted consent discussion be focused, similar to previously published models of "generic consent." 21 We suggest that a targeted discussion include why a genetic test is being offered, the possible outcomes (including variants of unknown significance), what the test can and cannot determine, the likelihood of a positive result, a brief overview of how results may affect management, and logistics including insurance issues. These basic concepts would provide patients adequate information to determine if they desire genetic testing and the future outcomes that may arise from such results.

Finally, to best provide result disclosure counseling for patients, genetics clinicians should either be proactively incorporated into any team ordering genetic testing or at least readily available as consultants. ${ }^{10}$ This will increase the likelihood that the genetics clinician is aware of what was discussed in a typical consent process and allows for potential training of medical team members by the genetics clinician. It also reduces the likely waiting period between a patient's learning of his/her genetic test result and the provision of TGC when needed.

\section{Limitations}

The snowball survey approach and qualitative methods used in this study were not chosen to reach consensus, but instead to identify issues that needed to be addressed in the development of the rubrics, including the relative importance of these issues. Further mixed methods research will be needed to determine whether the two rubrics meet the needs of providers and patients, and to assess their efficacy in clinical contexts.
Box 1 Reasons to consider referral to traditional genetic counseling (TGC) at time of consent

- Patient request

- Patient anxiety

- Patient has high chance of a positive predictive result (nondiagnostic)

- Patient has limited or mistaken information

- Patient has limited scientific, medical, or numerical literacy

- Patient is an adolescent participating in a genetic testing decision

- Provider feels uncertain about genetic test ordering or interpretation

\section{Reasons to consider brief communication with educational materials (if available)}

- Patient is knowledgeable about condition, perhaps already carrying a clinical diagnosis

- Patient has previously undergone traditional genetic counseling

- The patient has adequate literacy, and educational materials are available in the preferred language

\section{Conclusions}

CADRe has developed two rubrics that will produce recommendations for communicating consent and disclosure of genetic test results. This study's formative data validated that the indication and patient context for testing (e.g., knowledge, anxiety) is highly relevant to the communication approach, and therefore any rubric-based approach requires clinician engagement. Our workgroup is curating the list of 59 "medically actionable" genes recommended by the ACMG for the return of results in clinical sequencing (ACMG Secondary Findings v.2.0) (ref. 22). We anticipate that the CADRe rubrics, paired with communication recommendations, can help busy clinicians operationalize a consent and disclosure process in a manner that supports and empowers patients as genomic medicine becomes the norm.

\section{ELECTRONIC SUPPLEMENTARY MATERIAL}

The online version of this article (https://doi.org/10.1038/s41436018-0093-6) contains supplementary material, which is available to authorized users.

\section{ACKNOWLEDGEMENTS}

ClinGen is primarily funded by the National Human Genome Research Institute (NHGRI) through the following three grants: U41HG006834, U41HG009649, U41HG009650. The NHGRI Ethical, Legal and Social Implications Research Program provided supplemental funding to the CADRe Workgroup through grants U01HG007437 and U41HG009649. ClinGen also receives 
support for content curation from the Eunice Kennedy Shriver National Institute of Child Health and Human Development (NICHD), through the following three grants: U24HD093483, U24HD093486, U24HD093487. Thanks to Dave Kaufman, Erin Ramos, Nicole Lockhart, and Eric Tricou (who provide ex officio support to the CADRe committee), Alice Popejoy (for editorial comments), and Josie Pervola and Tacy Framhein (for assistance in recruitment and scheduling of the interviews and focus groups).

\section{DISCLOSURE}

Seema Jamal is an employee at GeneDx. Misha Raskin is an employee and stockholder at Helix, LLC. The other authors declare no conflict of interest.

\section{REFERENCES}

1. Hoskovec, JM, Bennett, RL, Carey ME, et al. Projecting the supply and demand for certified genetic counselors: a workforce study. J Genet Counsel 2017. 27:16-20. (2018).

2. Chambers CV, Axell-House DB, Mills G, et al. Primary care physicians' experience and confidence with genetic testing and perceived barriers to genomic medicine. J Fam Med. 2015;2:1024.

3. Bensend TA, Veach PM, Niendorf KB. What's the harm? Genetic counselor perceptions of adverse effects of genetics service provision by non-genetics professionals. J Genet Couns. 2014;23:48-63.

4. Brierley $\mathrm{KL}$, Blouch $\mathrm{E}$, Cogswell W, et al. Adverse events in cancer genetic testing: medical, ethical, legal, and financial implications. Cancer J. 2012;18:303-309.

5. Kurian AW, Li Y, Hamilton AS, et al. Gaps in Incorporating germline genetic testing into treatment decision-making for early-stage breast cancer. J Clin Oncol. 2017;35:2232-2239.

6. Miller CE, Krautscheid P, Baldwin EE, et al. Genetic counselor review of genetic test orders in a reference laboratory reduces unnecessary testing. Am J Med Genet A. 2014;164A:1094-1101.

7. Hughes KS. Genetic testing: what problem are we trying to solve? J Clin Oncol. 2017;35:3789-3791.
8. Childers CP, Childers KK, Maggard-Gibbons M, et al. National estimates of genetic testing in women with a history of breast or ovarian cancer. J Clin Oncol. 2017;35:3800-3806.

9. Ramos E, Haidle J. Genetic testing: multiple problems to solve. J Clin Oncol. 2018;36:518-519.

10. Randall LM, Pothuri B, Swisher EM, et al. Multi-disciplinary summit on genetics services for women with gynecologic cancers: a Society of Gynecologic Oncology white paper. Gynecol Oncol. 2017;146:217-224.

11. Etikan I, Alkassim R, Abubakar S. Comparison of snowball sampling and sequential sampling technique. Biom Biostat Int J. 2016;3:00055.

12. Dedoose [computer program]. Version 7.0.23. Los Angeles, CA: SocioCultural Research Consultants, LLC; 2016. http://www.dedoose. com. needs an access date

13. Reilly PR, Boshar MF, Holtzman SH. Ethical issues in genetic research: disclosure and informed consent. Nat Genet. 1997;15:16-20.

14. Geller G, Botkin JR, Green MJ, et al. Genetic testing for susceptibility to adult-onset cancer. The process and content of informed consent. JAMA. 1997;277:1467-1474.

15. Cohen SA, Nixon DM. A collaborative approach to cancer risk assessment services using genetic counselor extenders in a multi-system community hospital. Breast Cancer Res Treat. 2016;159:527-534.

16. Lieberman S, Lahad A, Tomer A, et al. Population screening for BRCA1/ BRCA2 mutations: lessons from qualitative analysis of the screening experience. Genet Med. 2017;19:628-634.

17. Lieberman S, Tomer A, Ben-Chetrit A, et al. Population screening for BRCA1/BRCA2 founder mutations in Ashkenazi Jews: proactive recruitment compared with self-referral. Genet Med. 2017;19:754-762.

18. Sutton EJ, Kullo IJ, Sharp RR Making pretest genomic counseling optional: lessons from the RAVE study [published online ahead of print February 1, 2018]. Genet Med. https://doi.org/10.1038/gim.2017.240.

19. Lea DH, Kaphingst KA, Bowen $D$, et al. Communicating genetic and genomic information: health literacy and numeracy considerations. Public Health Genomics. 2011;14:279-289.

20. Broady KM, Ormond KE, Topol EJ, et al. CS Predictors of adverse psychological reactions to receipt of direct-to-consumer genome-wide profiling results. J Community Genet 2017. 9:217-225.

21. Elias S, Annas GJ. Generic consent for genetic screening. N Engl J Med. 1994;330:1611-1613.

22. Kalia S, Adelman K, Bale $S$, et al. Recommendations for reporting of secondary findings in clinical exome and genome sequencing, 2016 update (ACMG SFv2.0): a policy statement of the American College of Medical Genetics and Genomics. Genet Med. 2017;19:249-255. 